\title{
钯基氧还原反应电极的构筑及其在水处理领域的研究进展
}

\author{
孙 猛 ${ }^{1,3}$ 李景虹 ${ }^{2, *}$ \\ ('中国科学院饮用水科学与技术重点实验室, 中国科学院生态环境研究中心, 北京 100085 ; \\ ${ }^{2}$ 清华大学化学系, 北京市微量分析与仪器重点实验室, 北京 100084; ${ }^{3}$ 中国科学院大学, 北京 100049)
}

\begin{abstract}
摘要: 发展氧气还原反应 $(\mathrm{ORR})$ 的二电子高效电催化剂一直是燃料电池领域的研究热点, 但针对具有二电子 还原特征且可应用于水处理领域电极材料的研究还处在起步阶段。本综述介绍了近年来二电子还原特征的贵 金属电极材料及其在电催化处理水中污染物的研究进展。在 $\mathrm{Fe}^{2+}$ 存在下, $\mathrm{Pd}$ 基电极材料催化氧气还原合成 $\mathrm{H}_{2} \mathrm{O}_{2}$, 间接催化氧化水中有机污染物, 实现有机物的矿化降解和水质的净化; $\mathrm{Pd}$ 基电极催化还原水中有机 污染物、无机盐等, 将其转化为低毒性、易处理产物并彻底去除; Pd 基催化电极的高效电子传导性能增强 了水中重金属离子的氧化/还原转化, 实现重金属去除。本综述展望了纳米电极材料在水处理应用的机遇与 发展方向。
\end{abstract}

关键词: 电催化; 氧气还原; 电极材料; 水处理

中图分类号: 0646

\section{Recent Progress on Palladium-Based Oxygen Reduction Reaction Electrodes for Water Treatment}

\author{
SUN Meng ${ }^{1,3} \quad$ LI Jing-Hong ${ }^{2, *}$ \\ ('Key Laboratory of Drinking Water Science and Technology, Research Center for Eco-Environmental Sciences, \\ Chinese Academy of Sciences, Beijing 100085, P. R. China; ${ }^{2}$ Department of Chemistry, Beijing Key Laboratory for \\ Microanalytical Methods and Instrumentation, Tsinghua University, Beijing 100084, P. R. China; \\ ${ }^{3}$ University of Chinese Academy of Sciences, Beijing 100049, P. R. China)
}

\begin{abstract}
Developing highly efficient electrode catalysts with the four-electron oxygen reduction pathway has remained a research hotspot in fuel cell research. However, the pursuit of novel electrode catalysts possessing the specific two-electron reduction route for water treatment is challenging. In this review, we focus on recent progress in electrocatalytic treatment of refractory pollutants in water by palladium $(\mathrm{Pd})$-based noble metal electrodes. We highlight: (i) the degradation and mineralization of organic pollutants through electrocatalytic oxidation derived from the combination of $\mathrm{Fe}^{2+}$ and $\mathrm{H}_{2} \mathrm{O}_{2}$, which can be in-situ synthesized by Pd based electrodes; (ii) electrocatalytic reduction transformation from toxic organic pollutants and inorganic salts to harmless products by $\mathrm{Pd}$-based electrodes; and (iii) removal of heavy metals by redox conversion via Pd-based electrodes. The future opportunities and prospects of applying noble metal nanocatalysts in water treatment are discussed.
\end{abstract}

Key Words: Electrocatalysis; Oxygen reduction; Electrode material; Water treatment

Received: July 18, 2016; Revised: September 16, 2016; Published online: September 19, 2016.

*Corresponding author. Email: jhli@mail.tsinghua.edu.cn; Tel: +86-10-62795290.

The project was supported by the National Key Research and Development Program of China (2016YFA0203101), National Key Basic Research Program of China (2013CB934004), National Natural Science Foundation of China (51572139), and Tsinghua University Initiative Scientific Research Program, China.

国家重点研究和发展项目(2016YFA0203101), 国家重点基础研究发展计划(2013CB934004), 国家自然科学基金(51572139)和清华大学计划科 研项目资助 


\section{1 引言}

进入二十一世纪以来, 能源与环境已成为全 球范围的热点问题。二者既相互依存，又相互制 约。人类生存环境的改善和社会的进步很大程度 上依赖于化石燃料的燃烧，而对能源无节制地滥 用却加剧了环境污染和温室效应。人类站在了 “能源换代” 和 “污染治理” 的交叉路口。如何开 发清洁、环境友好的新能源催化剂, 协同实现环 境污染物质的高效去除, 是新时代能源与环境技 术革新的重要动力和发展方向, 也是实现可持续 发展长远战略的根本保证。

氧气还原反应 $(\mathrm{ORR})$ 是氧气 $\left(\mathrm{O}_{2}\right)$ 在阴极表面直 接得到电子发生还原反应生成清洁水 $\left(\mathrm{H}_{2} \mathrm{O}\right)$ 的高效 能量转换过程, 在金属-空气电池以及醇类燃料电 池等领域已有广泛应用 1,2 。根据不同介质条件与阴 极材料性质, ORR 具有不同的动力学过程。在碱 性介质中, 吸附到电极表面的 $\mathrm{O}_{2}$ 可经过四电子过 程直接还原为 $\mathrm{OH}^{-}$, 也可通过二电子过程生成中间 价态的含氧吸附离子, 再进一步被还原为 $\mathrm{OH}^{-}$或直 接扩散到溶液中。在酸性介质中, $\mathrm{O}_{2}$ 既可以得到 四个电子一步还原为 $\mathrm{H}_{2} \mathrm{O}$, 还可以经过两步还原反 应过程, 即吸附到电极表面的 $\mathrm{O}_{2}$ 先得到二个电子 生成中间产物过氧化氢 $\left(\mathrm{H}_{2} \mathrm{O}_{2}\right), \mathrm{H}_{2} \mathrm{O}_{2}$ 再得到二个电 子进一步转化为 $\mathrm{H}_{2} \mathrm{O}^{3,4}$ 。酸性条件下, ORR 的动力 学反应过程如下:

$$
\begin{array}{ll}
\text { 总反应: } & \mathrm{O}_{2}+4 \mathrm{H}^{+}+4 \mathrm{e}^{-} \rightarrow 2 \mathrm{H}_{2} \mathrm{O} \\
\text { 分步反应: } & \mathrm{O}_{2} \rightarrow \mathrm{O}_{2}{ }^{*} \\
& \mathrm{O}_{2}{ }^{*}+\mathrm{H}^{+}+\mathrm{e}^{-} \rightarrow \mathrm{HOO}^{*} \\
\text { 二电子过程: } & \mathrm{HOO}^{*}+\mathrm{H}^{+}+\mathrm{e}^{-} \rightarrow \mathrm{H}_{2} \mathrm{O}_{2} \\
\text { 四电子过程: } & \mathrm{HOO}^{*} \rightarrow \mathrm{HO} \cdots \mathrm{O}^{*} \rightarrow \\
& \mathrm{HO}^{*}+\mathrm{O}^{*} \\
& \mathrm{HOO}^{*}+\mathrm{H}^{+} \rightarrow \mathrm{HOOH} \rightarrow \\
& \mathrm{HO} \cdots \mathrm{OH} \rightarrow 2 \mathrm{HO}^{*} \\
& \mathrm{O}^{*}+\mathrm{H}^{+} \rightarrow \mathrm{HO}^{*}
\end{array}
$$

$$
\mathrm{HO}^{*}+\mathrm{H}^{+}+\mathrm{e}^{-} \rightarrow \mathrm{H}_{2} \mathrm{O}
$$

可见, $\mathrm{O}-\mathrm{O}$ 键的断裂决定了 $\mathrm{ORR}$ 反应的最终 产物到底是 $\mathrm{H}_{2} \mathrm{O}$ 还是 $\mathrm{H}_{2} \mathrm{O}_{2}$ 。性能良好的 $\mathrm{ORR}$ 催化 剂显然需要与诸多含氧基团(例如 HOO*, $\mathrm{HO}^{*}$, $\mathrm{O}_{2}$ *等)有适当的反应结合能, 以突破热力学能垒而 发生相关反应。同时, 为满足高效能量转换要 求, 还应具备阻断 $\mathrm{O}-\mathrm{O}$ 键合并促进 $\mathrm{O}-\mathrm{H}$ 键形成 的能力, 以实现四电子传输过程。目前, 铂基催 化剂对 ORR 反应具有高活性和高稳定性, 显著降 低了 ORR 反应过电位, 是燃料电池工业最常用的 一类电极材料。由于铂资源稀缺、价格昂贵, 且 在反应过程中易发生 $\mathrm{CO}$ 及醇中毒效应导致失活等 问题, 限制了其应用化水平。理论研究也表明, Pt 对 $\mathrm{O}_{2}$ 的结合力过强而抑制了氧原子从其表面的脱 离, 阻断了四电子还原过程 ${ }^{5}$ 。研发成本低、催化 活性稳定、具有高选择性动力学过程的氧还原电 极材料一直是能源研究领域的热点。

在追求 ORR 的四电子高效能量转化过程中, 人们却忽视了二电子转移途径在电化学处理水体 有毒有害物质中的作用。近年来, 以绿色、高效 为典型特征的电化学水处理技术利用电极反应及 其相关过程, 通过直接或间接的氧化还原、凝聚 絮凝、吸附降解和协同转化等综合作用, 在含有 难降解有机物、重金属、无机盐类、胶体颗粒物 等污染物的特种废水处理中独具优势, 应用越来 越广泛。例如, 在电化学催化氧化降解有机污染 物过程中, 铁板阳极失去电子产生 $\mathrm{Fe}^{2+}$, 同时阴极 表面发生氧气还原反应生成少量 $\mathrm{H}_{2} \mathrm{O}_{2}$, 二者发生 间接催化反应生成强氧化性羟基自由基, 可无选 择性与水中有害有机污染物发生作用, 使其矿化 降解为 $\mathrm{H}_{2} \mathrm{O}$ 和 $\mathrm{CO}_{2}$, 达到水体净化的目的。这显示 了原位电催化技术在水处理领域的重要应用价 值。与其他外源投加 $\mathrm{H}_{2} \mathrm{O}_{2}$ 的水处理技术相比较, 原位电催化技术既降低了化学品在储存、运输、

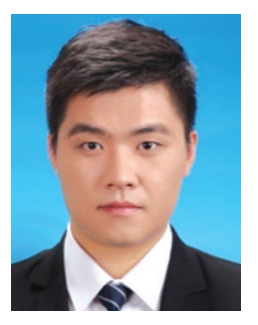

孙猛, 1986 年生。获得中国科学 院生态环境研究中心环境工程博士 学位。现于耶鲁大学从事博士后研 究。研究领域: 新型电极材料的合 成及其在能源与环境领域中的应 用、电化学水质净化技术与原理研

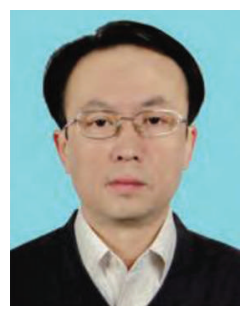
李景虹, 1967年生。清华大学化学 系教授、博士生导师。主讲课程: 仪器分析、化学与社会。研究领 域: 电分析化学、生物传感分析、 单细胞分析、纳米电化学。

究、膜水处理技术。 
投配过程中的成本，也大大提升了其在催化反应 中的利用效率, 避免了物质浪费并减缓了二次污 染, 极大地提高了水处理效率, 符合发展能源节 约、资源回收、有害物质消除等多目标协同一体 的新型水处理理念。但是, 目前常用的电化学水 处理阴极材料原位合成 $\mathrm{H}_{2} \mathrm{O}_{2}$ 的效率亟待提高。诸 多研究采用在反应器中曝气或者其他增氧方式 ${ }^{6}$, 强化电化学阴极直接产生 $\mathrm{H}_{2} \mathrm{O}_{2}$ 的效率, 增加了水 处理成本, 同时却收效甚微。此外, 拥有大比表 面积的气体扩散电极、活性炭纤维等新型电极材 料也被用作阴极应用于电催化水处理技术中, 但 其对原位合成 $\mathrm{H}_{2} \mathrm{O}_{2}$ 的提升作用并不显著。基于特 定 ORR 的二电子过程的电催化原位合成 $\mathrm{H}_{2} \mathrm{O}_{2}$ 的电 催化研究, 无论对于氧还原基础理论的探索还是 其相关环境水处理技术的应用都具有重要意义。

本综述以钯 $(\mathrm{Pd})$ 基催化电极为例, 结合其 $\mathrm{ORR}$ 催化特征, 重点介绍了近年来 Pd 电极材料在电化 学水处理领域应用的研究进展, 包括电催化氧化 有机物去除, 电催化还原有机物、无机盐, 电催 化协同重金属氧化还原转化等。对 $\mathrm{Pd}$ 基电极形 貌、结构、性能等进行比较, 以期揭示结构一性能
之间的潜在关系, 为电催化在能源与环境领域的 交叉研究提供理论基础。

\section{$2 P d$ 基催化剂结构与电催化性能}

贵金属 Pd 元素的地球储量是 Pt 的 3 倍, 价格 比 $\mathrm{Pt}$ 低廉。同时, $\mathrm{Pd}$ 又被公认与氢元素具有最强 的亲和力, 因此其在氢化反应、氢能的提纯净化 与储存等领域都表现出了超越 $\mathrm{Pt}$ 的催化性能, 显 现出巨大的应用前景 ${ }^{7}$ 。此外, 相比其它类似贵金 属及非金属催化剂, Pd丰富的晶体结构与形貌特 征, 不仅决定了其多效催化活性, 而且其催化性 能不容小㱆。近年来, 对 Pd 纳米材料形貌、结 构、尺寸的调控及其性能进行了系统研究 ${ }^{8}$ 。如图 1 所示, 金属 Pd 的面心立方结构决定了其具有形成 多种几何结构的潜质, 可以通过热力学或动力学 因素对其形成过程加以调控。研究表明 Pd 晶种的 不同晶体结构决定了其最终生长的形貌和结构 ${ }^{9,10}$ 。 例如, 可由暴露出(111)和(100)晶面的 Pd 晶种制备 八面体、立方八面体或立方体, 而暴露晶面的种类 和比例显著影响 Pd纳米结构的生成。Yarulin等 ${ }^{11}$ 发 现 $\operatorname{Pd}(111)$ 面暴露较多的八面体纳米催化剂的电石

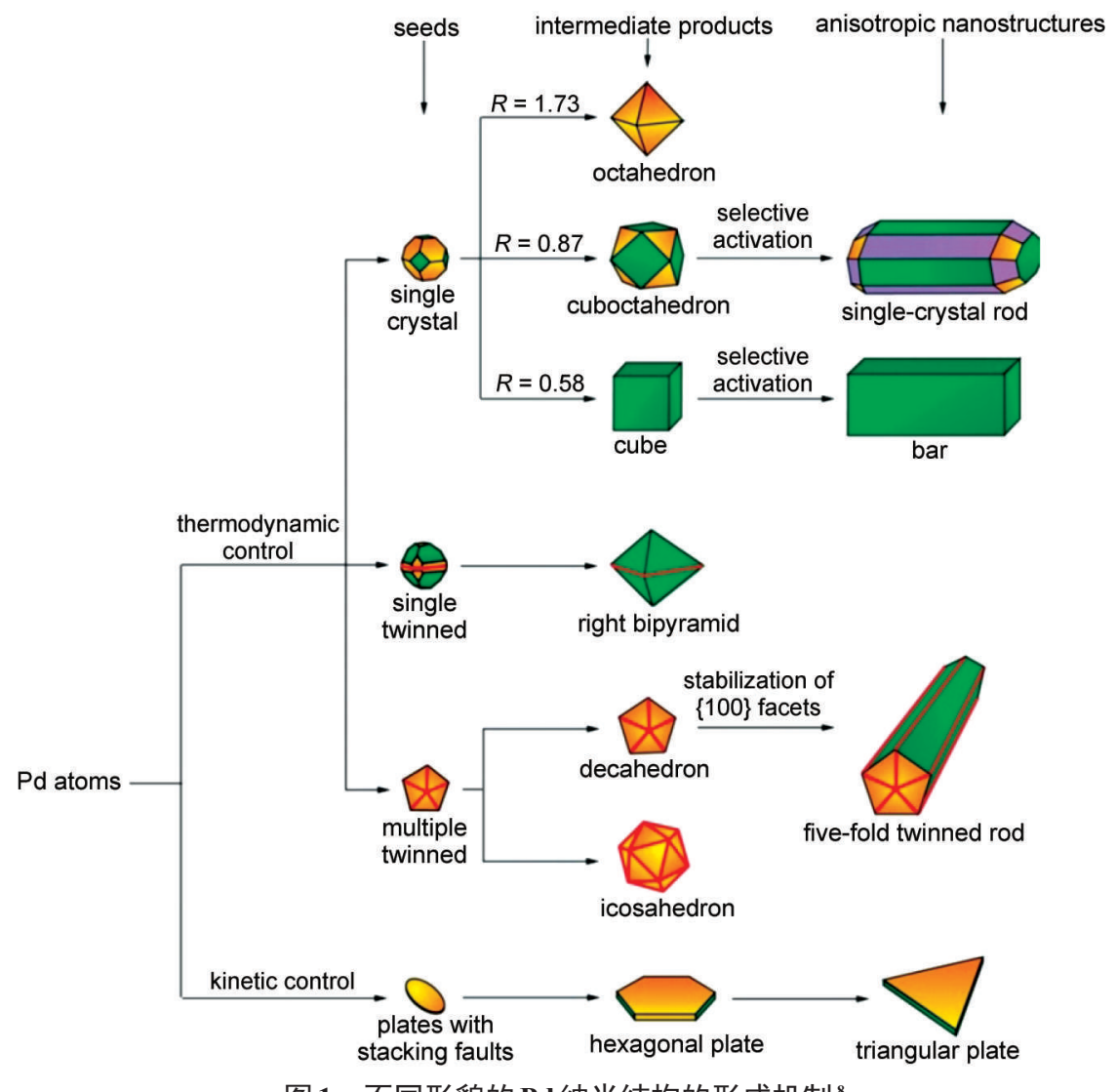

图 1 不同形貌的 Pd 纳米结构的形成机制 ${ }^{8}$

Fig.1 Reaction pathways that lead to Pd nanostructures with different morphologies ${ }^{8}$ 
气产氢性能比 $\mathrm{Pd}(100)$ 高出近 1.5 倍, 证实了 $\mathrm{Pd}$ 金 属晶体结构与性能之间的内在联系。Jin 等 ${ }^{12}$ 发 现, 调节 Pd 表面(100)和(111)晶面暴露比例, 可形 成多种形貌的 Pd 纳米颗粒, 诸如立方体、无棱角 的立方体、八面体和无棱角的八面体等, 这些 $\mathrm{Pd}$ 纳米颗粒的电催化性能差别显著。 Pd(111) 和 Pd (100)有利于加酸的催化氧化和阳极过电位的提 升。除上述多面体结构外, Pd螺旋、尖䢃状等 ${ }^{13-15}$ 特殊形貌结构的研究也丰富了 $\mathrm{Pd}$ 晶体的生长特 征, 为多领域应用奠定了基础(图 2)。此外, Pd 及 催化剂颗粒的平均粒径也显著影响电催化活性及 选择性。原子间结合力、分子间桥联作用决定了 纳米团簇的尺寸, 进而影响纳米颗粒的诸多理化 性质。 $\mathrm{Jin}$ 等 ${ }^{12}$ 在 $\mathrm{ZnO}$ 的纳米线上分别合成了 $6 、 10$ 和 $18 \mathrm{~nm}$ Pd 纳米颗粒。研究发现, 随着 Pd颗粒尺 寸的降低, $\mathrm{CO}$ 氧化速率明显提高。小尺寸 $\mathrm{Pd}$ 基催 化剂在氢能储存、有机酸氧化、氧气还原等领域 发挥了巨大优势 ${ }^{16-19}$ 。

\section{$2.1 P d$ 纳米催化剂合成方法研究}

目前已发展的合成方法包括电沉积法 ${ }^{15}$ 、引极 真空喷镀涂覆法 ${ }^{18,20}$ 、离子/电子束诱导沉积法 ${ }^{21,22}$ 、 激光烧蚀法 ${ }^{23,24}$ 、水热法 ${ }^{25-27}$ 、高温㷽烧法 ${ }^{28}$ 、化学 还原 ${ }^{29-31}$ 等。喷镀涂覆法、离子/电子束诱导沉积法 和激光烧蚀法皆属于物理合成方法。喷镀涂覆法 是在真空或氩气气氛下, 通过电场作用使熔融态 金属前驱体以一定的流量涂覆在铂片或其他基底 材料上。喷涂时间决定了形成金属片层的厚度 ${ }^{32}$ 。 这种方法可以较好地控制金属片层的厚度、形 貌, 调节纳米团簇尺寸。与精于构建二维平面结
构的涂覆法相比，离子/电子束诱导沉积法则在合 成金属的三维结构上更具优势。如图 3 所示, 金属 前驱体单体在电子束的诱导下在基底材料上实现 排列、拼接和组装形成三维立体结构, 例如纳米 线和特定图案等 ${ }^{22,33,34}$ 。激光蚀刻法是利用激光激发 金属等离子体向靶向基底沉积的过程, 其具备操 作简单、合成纳米颗粒分散性好和易于多种组分 合成等优势。Giuffrida 等 ${ }^{35}$ 选择不同波长的激发光 源对 $2 \mathrm{~cm}^{2} \mathrm{Pd}$ 纳米片进行蚀刻, 并发现近红外波段 的激光较可见光更有利于蚀刻过程。近年来, 激 光蚀刻的应用环境介质条件也成为该领域研究热 点。如图 3 所示, Mortazavi 等 ${ }^{36}$ 在去离子水中实现 了两种激光光源对 $\mathrm{Pd}$ 蚀刻。显然, 液体中的刻蚀 过程有利于纳米颗粒的稳定分散, 但蚀刻后纳米 颗粒的尺寸及形貌也受到溶剂性质、激光波长及 蚀刻时间等因素的影响。相对于物理法合成 Pd 纳 米材料, 化学还原与水热还原法应用更为广 泛 $35,37,38$ 。与化学还原相比, 水热法一般在高温高压 条件下一步实现含 Pd 溶液的还原, 形成多种 Pd 纳 米结构。研究表明, 一方面在水热过程中, 还原 剂诸如加酸、甲醛、乙二醇、水合肼、柠檬酸、 抗坏血酸等会显著影响了合成 Pd 纳米颗粒的尺寸 和形貌; 另一方面, 表现活性剂及高分子聚合物 等助剂的使用, 也会决定产物 Pd 纳米颗粒的结 构。例如, Lee 等 ${ }^{39}$ 借助表面活性剂十六烷基三甲 基铵硝酸盐(CTAC)制备了八面体 $\mathrm{Au} @ \mathrm{Pd}$ 核壳结 构。 $\mathrm{Xu}$ 等 ${ }^{40}$ 利用一种弱还原剂对苯二酚合成了 $\mathrm{Pd}$ 包裹 $\mathrm{Au}$ 核的花状核壳结构, 该结构高效实现了对 碘代苯的催化转化。电沉积法是另一种应用较广

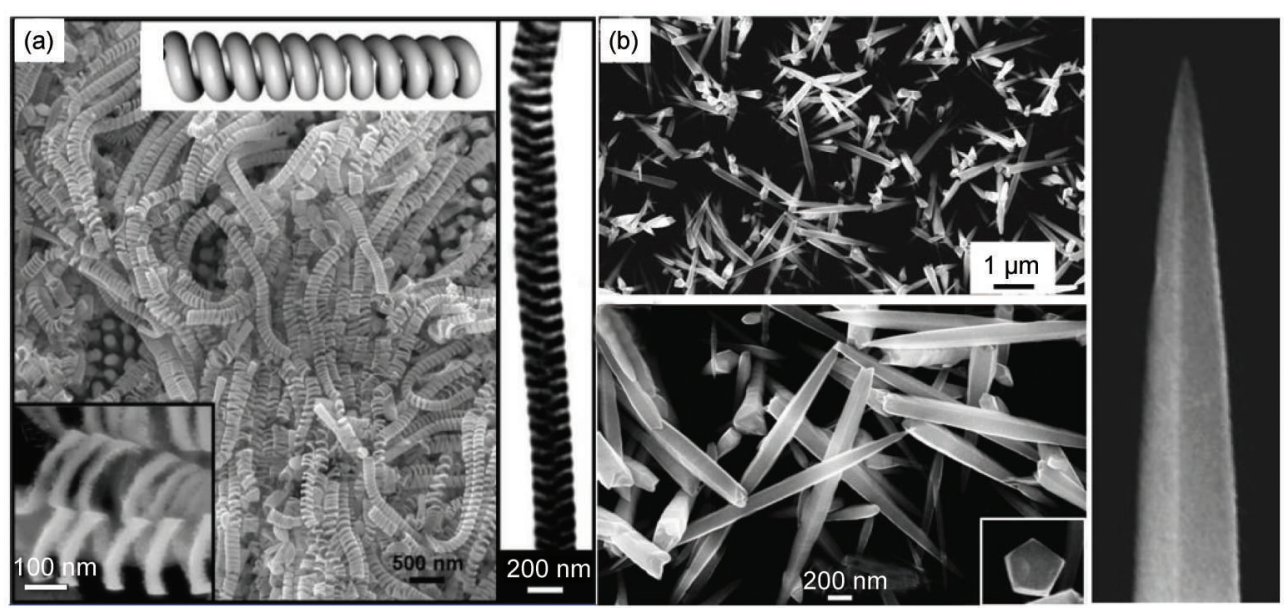

图 2 (a)弹簧状纳米 Pd 的扫描电镜(SEM) 与透射电镜(TEM)组图 ${ }^{13}$; (b) 尖䢃状 Pd 纳米棒的 SEM 组图 ${ }^{14}$

Fig.2 (a) Scanning electron microscopy (SEM) and transmission electron microscopy (TEM) images of large quantity of Pd handsprings ${ }^{13}$; (b) SEM images of Pd nanorods ${ }^{14}$ 


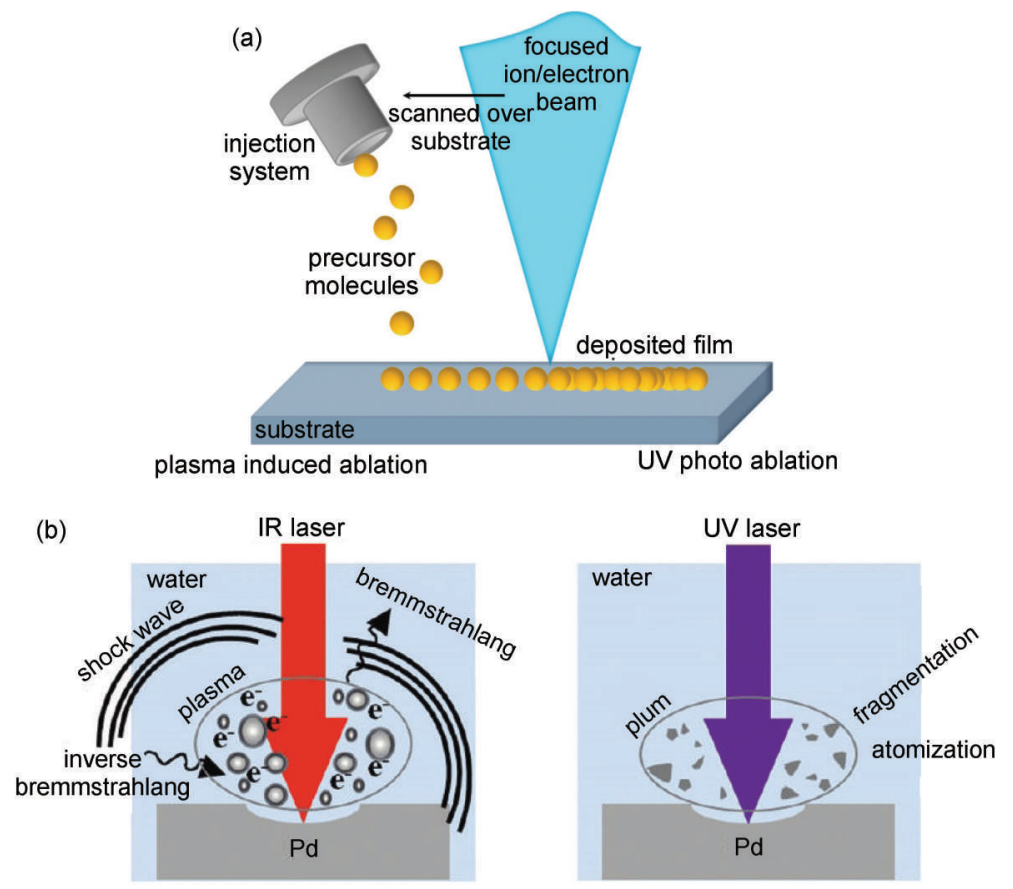

图 3 (a)离子/电子束诱导沉积法示意图 ${ }^{8}$; (b) 可见光及紫外光诱导 Pd 纳米颗粒生长机制示意图 ${ }^{36}$

Fig.3 (a) Schematic representation of focused ion/electron beam induced deposition techniques ${ }^{8}$;

(b) effect of laser and UV on the structural formation of palladium nanoparticles ${ }^{36}$

泛的易于制备 Pd 纳米管、纳米球、纳米棒阵列等 形貌的方法 ${ }^{41-44}$ 。一般来说, 电沉积法是利用双电 极或三电极体系将电解质中的 Pd 还原在阴极上。 因此, 还原电压、电流及还原时间决定了形成 Pd 颗粒的形貌和尺寸。

\section{$2.2 P d$ 催化性能、机制研究}

$P d$ 催化应用范围较为广泛, 如能源催化领域 中的直接高效氧化甲醇 ${ }^{45}$ 、甲酸等燃料 ${ }^{46}$, 可用作 理想的清洁能源电池阴极材料。还可应用于产 氢、气体传感器和电化学生物传感器等 ${ }^{47}$ 。 Pd 在电 催化水处理中可作为直接阴极、颗粒电极、填充 式感应电极、电催化剂等使用, 获得较高的水处 理效率。此外, 氧气在 Pd 基催化剂表面发生还原

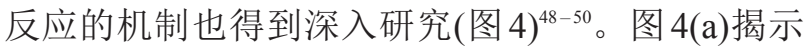
了 $\mathrm{O}_{2}$ 在 $\mathrm{Pd}$ 基合金催化剂(001)活性面被快速催化经 过两步形成 $\mathrm{H}_{2} \mathrm{O}_{2}$ 的分解过程。在 $\mathrm{Pd}$ 基催化剂表面 $\mathrm{O}_{2}$ 的还原反应中, 在平衡电位 $\left(U_{\mathrm{O}_{2} / \mathrm{H}_{2} \mathrm{O}_{2}}=0.7 \mathrm{~V}\right)$ 下催 化剂对中间产物 HOO*的吸附是热力学中性即自发 进行的, 保证该初始反应的理论过电位恰好为 0 , 其与 $\mathrm{HOO}^{*}$ 稍强或过弱的结合力将导致多余的过电 位产生或催化活性降低。因此, 可根据不同贵金 属与 $\mathrm{Pd}$ 基催化剂与 $\mathrm{HOO}^{*}$ 的结合能变化 $\left(\Delta G_{\mathrm{HOO}^{*}}\right)$ 的 火山图来预测其催化生成过氧化氢的活性(见图 4 (b)和 4(c))。由图 4(c) 可见, Pd 基催化剂催化合成 $\mathrm{H}_{2} \mathrm{O}_{2}$ 活性较强, 依次为 $\mathrm{Pt}>\mathrm{Ag}>\mathrm{Cu}>\mathrm{Au}$ 。此外, 电催化合成 $\mathrm{H}_{2} \mathrm{O}_{2}$ 的选择性取决于催化剂对 $\mathrm{O}-\mathrm{O}$ 键 的保护能力, 以防止 $\mathrm{H}_{2} \mathrm{O}$ 的形成 $\left(U_{\mathrm{O}_{2} \mathrm{H}_{2} \mathrm{O}}=1.23 \mathrm{~V}\right)$ 。 诸多密度泛函理论计算与实验结果相互印证了 $\mathrm{Pd}$ 催化剂具有较强的抑制 $\mathrm{O}-\mathrm{O}$ 键断裂的能力, 使其 不能解离为单个氧原子, 促使 $\mathrm{HOO}^{*}$ 得到一个电子 与质子 $\mathrm{H}$ 结合最终还原为 $\mathrm{H}_{2} \mathrm{O}_{2}{ }^{51,52}$ 。再者, $\mathrm{Pd}$ 基催 化剂的高催化氢化性能还可在表面将阳极电解水 产生的 $\mathrm{H}_{2}$ 解离为 2 个原子 $\mathrm{H}$, 原子 $\mathrm{H}$ 的强还原性有 助于其与 $\mathrm{HOO} *$ 结合生成 $\mathrm{H}_{2} \mathrm{O}_{2}{ }^{53,54}$ 。可见, $\mathrm{Pd}$ 基催 化剂的催化特征决定了其催化合成 $\mathrm{H}_{2} \mathrm{O}_{2}$ 的高活性 与专一性。这种优势在报道的 $\mathrm{PdAu}^{55,56} 、 \mathrm{PdHg}$ 及 CuPd 合金催化剂中更为显著 $55,57-59$ 。

\section{$3 \mathrm{Pd}$ 电催化反应在电催化水处理中的应用}

\section{1 电催化氧化降解水中有机污染物}

Yuan 课题组 ${ }^{60}$ 较早开展了基于 Pd 电催化降解 水体有机污染物的研究, 涉及模拟废水及地下水 的污染治理及污染物降解机制过程研究。他们首 先合成了 $\mathrm{Pd} / \mathrm{C}$ 颗粒电极填充于阴、阳两极之间作 为感应电极, 用于原位电催化氧化降解罗丹明 $\mathrm{B}$ 废 水(见图 5)。如图 5(a)所示, 他们构建了双电解池 
(a)

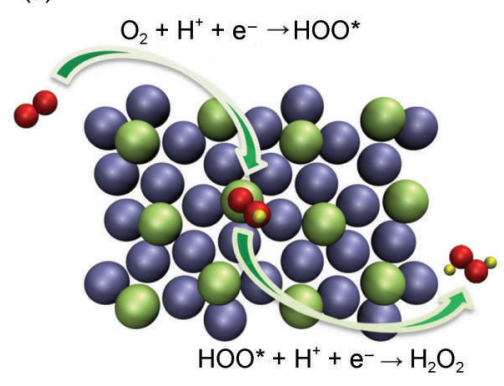

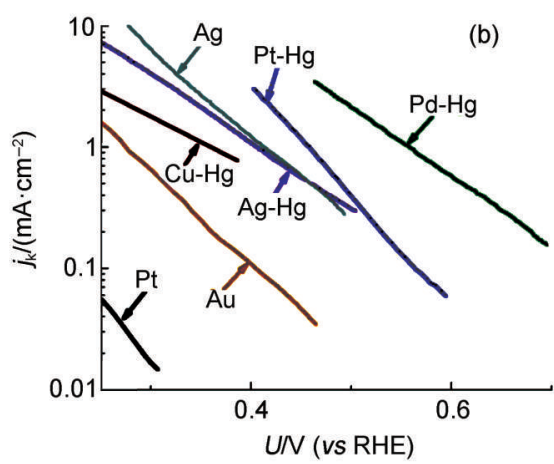

(b)

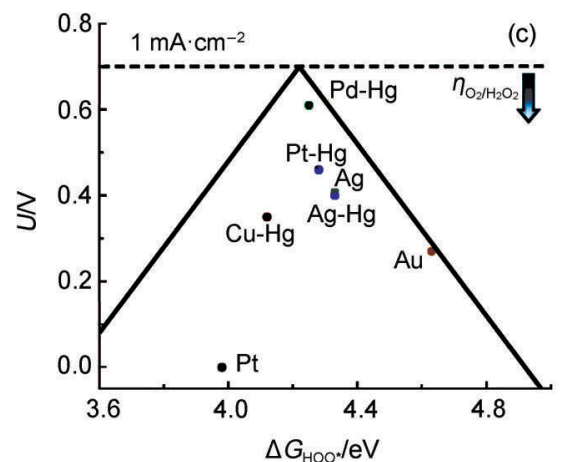

图 4 (a) Pd 基贵金属催化剂氧气还原合成 $\mathrm{H}_{2} \mathrm{O}_{2}$ 机制; (b)贵金属及其合金催化剂合成 $\mathrm{H}_{2} \mathrm{O}_{2}$ 动力学电流与电位关系; (c)贵金属及其合金催化剂合成 $\mathrm{H}_{2} \mathrm{O}_{2}$ 热力学火山图 ${ }^{59}$

Fig.4 (a) Schematic representation of oxygen reduction to $\mathrm{H}_{2} \mathrm{O}_{2}$ on Pd based catalysts; (b) partial kinetic current density to $\mathrm{H}_{2} \mathrm{O}_{2}$ as a function of the applied potential, corrected for mass transport losses; (c) thermodynamics volcanic figure of $\mathrm{H}_{2} \mathrm{O}_{2}$ synthesis on noble metal and their alloys ${ }^{59}$

For Fig.(c), potential required to reach $1 \mathrm{~mA} \cdot \mathrm{cm}^{-2}$ of kinetic current density to $\mathrm{H}_{2} \mathrm{O}_{2}$ on polycrystalline catalysts as a function of the calculated HOO* binding energy. The solid lines represent the theoretical Sabatier volcano.

三电极电催化体系, 实现了原位生成 $\mathrm{H}_{2} \mathrm{O}_{2} 、 \mathrm{pH}$ 自 调节, $\mathrm{H}_{2}$ 和 $\mathrm{O}_{2}$ 的持续供给等功能。结果表明, 在 $\mathrm{pH}=2$, 电流 $100 \mathrm{~mA}$ 时, $\mathrm{Pd} / \mathrm{C}$ 颗粒电极可高效电 催化 $\mathrm{O}_{2}$ 还原生成 $\mathrm{H}_{2} \mathrm{O}_{2}$, 致使电催化氧化体系中的 $\mathrm{H}_{2} \mathrm{O}_{2}$ 在 $120 \mathrm{~min}$ 内的浓度可达到 $53.1 \mathrm{mg} \cdot \mathrm{L}^{-1}$, 该浓 度足以与外源加入的二价铁离子发生催化反应生 成羟基自由基(见反应式(9)), 导致罗丹明 $\mathrm{B}$ 以近似 于拟一级动力学过程被快速去除。此外, 图 5(b) 和 5(c) 证明了 $\mathrm{Pd} / \mathrm{C}$ 颗粒电极持续稳定电催化合成 $\mathrm{H}_{2} \mathrm{O}_{2}$ 和循环降解罗丹明 $\mathrm{B}$ 的能力。这个工作为 $\mathrm{Pd}$ 基催 化剂在电催化水处理领域的应用研究提供了启 示。在此基础上, 他们还合成了可磁性回收的高 效 $\mathrm{Pd} / \mathrm{Fe}_{3} \mathrm{O}_{4}$ 催化颗粒电极, 其高效可循环利用的能 力显著提升了其应用潜质 ${ }^{61}$ 。该催化电极可以催化 合成充足的 $\mathrm{H}_{2} \mathrm{O}_{2}, 60 \mathrm{~min}$ 内即可使 $98 \%$ 的苯酚矿 化降解。从图 5(d) 可以看出, 磁性基底 $\mathrm{Fe}_{3} \mathrm{O}_{4}$ 不仅 为 $\mathrm{Pd}$ 金属提供了稳定的载体, 又作为铁源提供铁 离子以保证催化氧化反应的进行。另外, $\mathrm{Pd}$ 一方 面具有催化 $\mathrm{O}_{2}$ 还原为 $\mathrm{H}_{2} \mathrm{O}_{2}$ 的功能, 另一方面其表 面形成的强还原性的氢原子可将 $\mathrm{Fe}^{3+}$ 还原为 $\mathrm{Fe}^{2+}$, 保持催化氧化反应的效率。

$$
\mathrm{H}_{2} \mathrm{O}_{2}+\mathrm{Fe}^{2+}+\mathrm{H}^{+} \rightarrow \mathrm{Fe}^{3+}+\cdot \mathrm{OH}+\mathrm{H}_{2} \mathrm{O}
$$

Sun 等 ${ }^{63}$ 通过浸渍还原方法合成了低负载量 金、钯双金属合金负载的碳纳米管催化电极, 并 将其作为感应电极分散于阴、阳极间, 高效催化 降解水中有机污染物 (图 6)。该 AuPd/CNTs 电极与 以往同类型电极相比, 除了具有更好的电催化原 位合成 $\mathrm{H}_{2} \mathrm{O}_{2}$ 和催化去除有机污物的能力, 其还具
备 $\mathrm{Fe}^{2+}$ 还原再生能力, 大大提升了二价铁离子的利 用效率, $37 \mathrm{~min}$ 内即可实现有机物 100\%矿化去 除, 其机制如图 6(e)所示。合金结构强化了晶体结 构稳定性, 保护了合金表面活性(111)晶面; 交错 的 CNTs 构成三维立体结构为 AuPd 合金提供了有 效的基底载体, 保证了物质传质的同时也避免了 合金在催化过程中团聚失活。该研究首次引入 AuPd 合金基催化电极应用于电催化水处理技术 中, 并取得了对水中多种有机污染物稳定可靠的 去除效果, 展现了 Pd基合金催化电极在水中有机 污染物降解消除领域的应用前景。基于此, Qin 等 ${ }^{64}$ 又采用化学还原法制备了可磁性回收 $\mathrm{AuPd} /$ $\mathrm{Fe}_{3} \mathrm{O}_{4}$ 催化电极, 协同实现了电极原位合成 $\mathrm{H}_{2} \mathrm{O}_{2}$ 和 $\mathrm{Fe}^{2+}$ 高效自给, 并对典型 1-丁基-3-甲基咪唑型离子 液体具有较好的催化降解效果。研究揭示基于 $\mathrm{AuPd} / \mathrm{Fe}_{3} \mathrm{O}_{4}$ 电极的电催化氧化体系与阳极芬顿氧化 模型(AFT)相似, 催化效率与溶解 $\mathrm{Fe}^{2+}$ 浓度显著相 关。该催化氧化体系展现了稳定的羟基自由基生 成效率, 磁性回收的便利使得 $\mathrm{AuPd} / \mathrm{Fe}_{3} \mathrm{O}_{4}$ 电极在 废水有机物催化降解处理中具有优势。

对于地下水中有机污染物的转化和消减, $\mathrm{Pd}$ 基催化电极也发挥了重要作用。Liao 等 ${ }^{65}$ 巧妙地利 用了地下水迁移的时空特性, 在污染晕的上游、 污染源及污染晕下游依次设立产氧阳极、析氢阴 极、Pd催化颗粒电极填充区域和调 $\mathrm{pH}$ 阴极的三电 极感应电催化系统, 有效实现了对地下水中甲基 叔丁醚(MTBE)的降解去除。如图 7(a)所示, Pd 催 化电极的填充区域发挥了其原位氧气催化还原功 

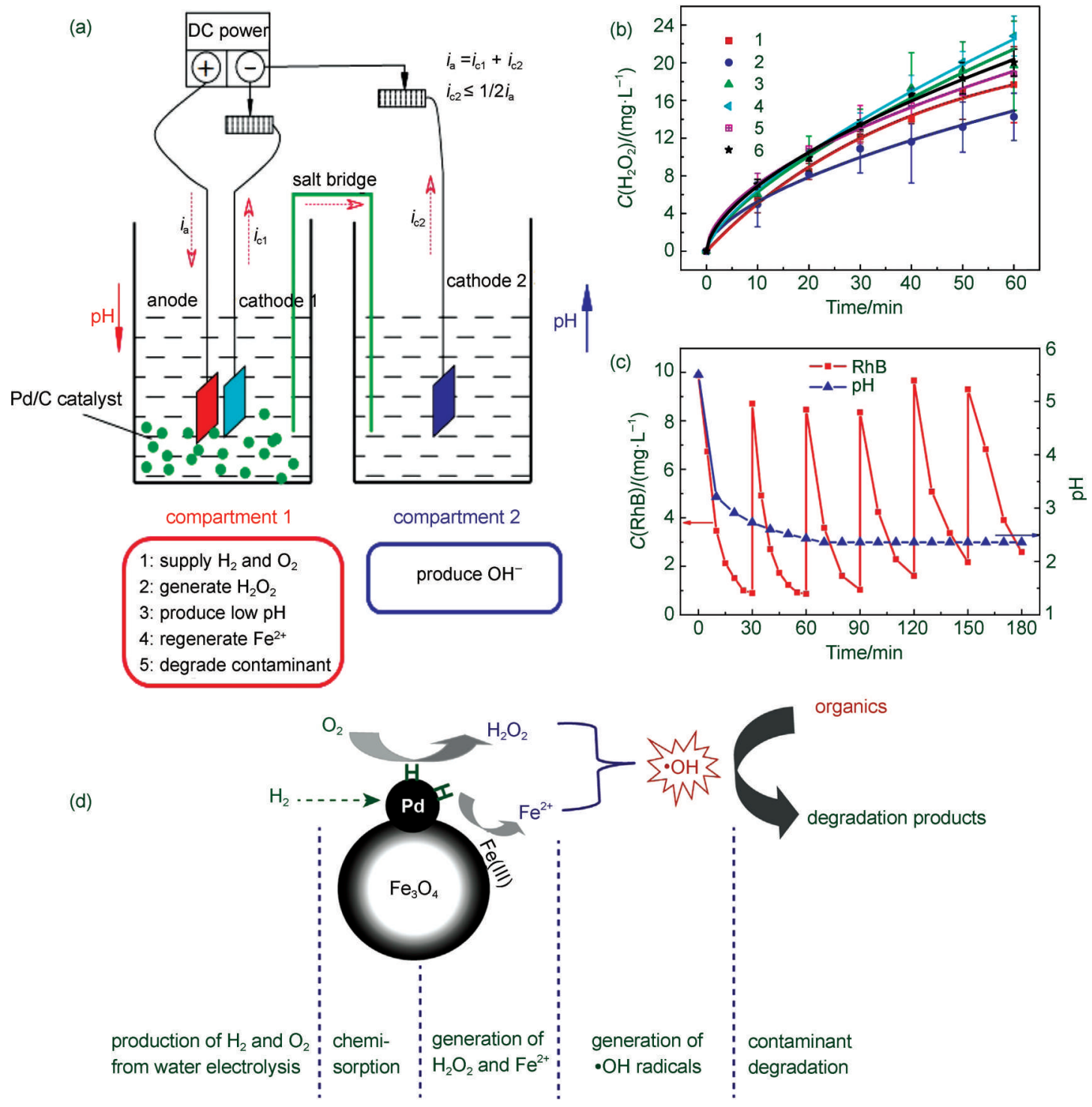

图 5 (a) $\mathrm{Pd} / \mathrm{C}$ 颗粒电极电催化氧化降解罗丹明 B 废水机制图; (b) $\mathrm{Pd} / \mathrm{C}$ 颗粒电极循环电催化合成 $\mathrm{H}_{2} \mathrm{O}_{2}$ 的浓度变化;

(c) $\mathrm{Pd} / \mathrm{C}$ 颗粒电极循环电催化降解罗丹明 B 废水效率 ${ }^{62}$; (d) $\mathrm{Pd} / \mathrm{Fe}_{3} \mathrm{O}_{4}$ 颗粒电极电催化氧化降解水中苯酚机制 ${ }^{61}$

Fig.5 (a) Scheme of efficient electrocatalytic oxidation degradation of $\mathrm{RhB}$ using $\mathrm{Pd} / \mathrm{C}$ particle electrodes; (b) repeated experiments on the electrocatalytic generation of $\mathrm{H}_{2} \mathrm{O}_{2}$ and (c) the degradation of $\mathrm{RhB}$ by $\mathrm{Pd} / \mathrm{C}$ particle electrodes ${ }^{62}$;

(d) proposed mechanisms on electrocatalytic oxidation degradation of phenol by $\mathrm{Pd} / \mathrm{Fe}_{3} \mathrm{O}_{4}$ particle electrodes $^{61}$

能, 促使生成充足的 $\mathrm{H}_{2} \mathrm{O}_{2}$, 与阳极电解产生的 $\mathrm{Fe}^{2+}$ 在适宜 $\mathrm{pH}$ 条件下发生催化氧化反应，可在长达 20 天内保持约 70\%的甲基叔丁醚矿化率。这一实验 设计精妙地根据地下水运移和电极反应特征，构 建不同的功能区域使有机污染物去除, 还避免了 地下水的二次污染。Yuan 等 ${ }^{62}$ 又完成了对模拟地下 水中优先污染物三氯乙烯(TCE)的去除。如图 7(b) 所示, 该去除过程主要包括 Pd 对 TCE 的直接氢化 还原作用以及在 $\mathrm{Fe}^{2+}$ 存在下对 $\mathrm{TCE}$ 的间接电催化氧 化降解。研究表明, 协同催化氧化与催化还原作 用可使初始浓度 $396 \mu \mathrm{mol} \cdot \mathrm{L}^{-1}$ 的 TCE 在 $80 \mathrm{~min}$ 内
去除 $94.9 \%$, 其中 $84 \%$ 是被催化产生的 $\mathrm{HO}$-氧化去 除的, 电催化氧化作用对 TCE 的去除更为高效。 除了 $\mathrm{HO}$-氧化, 还揭示了 $\mathrm{SO}_{4}^{-} \cdot$ 对于 $\mathrm{TCE}$ 去除的贡 献。他们在地下水污染区域内构建了铁阳极与阴 极的双电极体系, 辅助添加过硫酸盐使其与阳极 溶解的 $\mathrm{Fe}^{2+}$ 反应生成活性 $\mathrm{SO}_{4}^{-} \cdot$, 以高效氧化降解 $\mathrm{TCE}$ (如图 7(c) 所示) ${ }^{66}$ 。过硫酸盐的加入不仅强化了 强氧化自由基 $\mathrm{SO}_{4}^{-}$的形成, 也参与了地下环境中 铁物质间的氧化还原转化, 降低铁的二次污染风 险, 为地下水中 TCE 污染的电催化处理提供了更 多选择。制备了 $\mathrm{Pd} / \mathrm{Al}_{2} \mathrm{O}_{3}$ 颗粒作为填充式电极, 在 

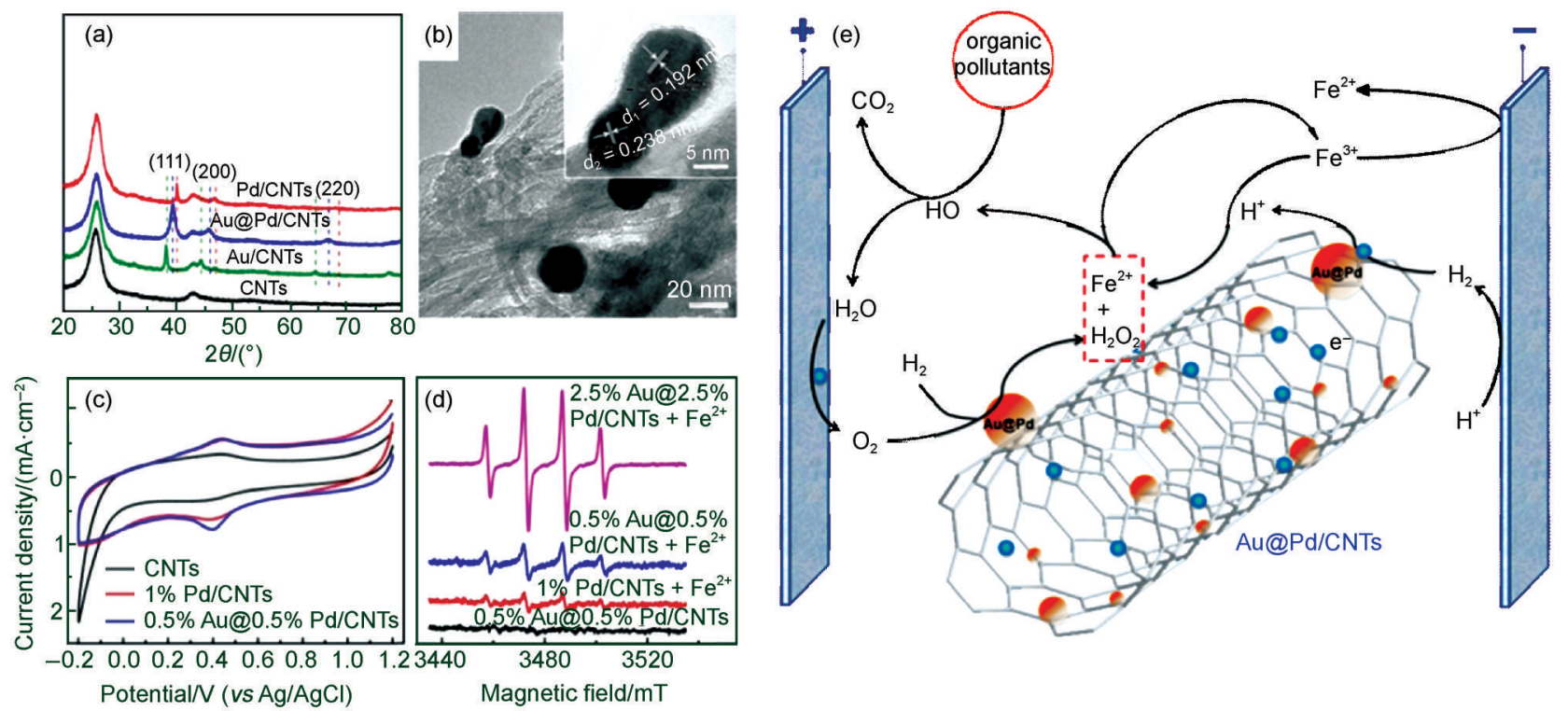

图 6 (a) $\mathrm{CNTs}, \mathrm{Pd} / \mathrm{CNTs}, \mathrm{Au} / \mathrm{CNTs}$ 和 $1 \% \mathrm{Au}_{0.5} \mathrm{Pd}_{0.5} / \mathrm{CNTs}$ 的 $\mathrm{X}$ 射线衍射(XRD)图; (b) $\mathrm{Au}_{0.5} \mathrm{Pd}_{0.5} / \mathrm{CNTs}$ 透射电镜图, 内插图为 局部高分辨透射图片；(c) CNTs, Pd/CNTs 和 $\mathrm{Au}_{0.5} \mathrm{Pd}_{0.5} / \mathrm{CNTs}$ 电极的循环伏安曲线，扫描速度： $50 \mathrm{mV} \cdot \mathrm{s}^{-1}$;

(d)电催化过程中的自由基 ESR 谱图; (e) $\mathrm{Au}_{0.5} \mathrm{Pd}_{0.5} / \mathrm{CNTs}$ 电催化降解水中有机污染物机制 ${ }^{63}$

Fig.6 (a) X-ray diffraction (XRD) patterns of CNTs, Pd/CNTs, $\mathrm{Au} / \mathrm{CNTs}$, and $1 \% \mathrm{Au}_{0.5} \mathrm{Pd}_{0.5} / \mathrm{CNTs}$; (b) TEM images of $\mathrm{Au}_{0.5} \mathrm{Pd}_{0.5} / \mathrm{CNTs}$, the inset shows its high resolution transmission electron microscopy (HRTEM) image; (c) $\mathrm{CV}$ curves of $\mathrm{CNTs}, \mathrm{Pd} / \mathrm{CNTs}$, and $\mathrm{Au}_{0.5} \mathrm{Pd}_{0.5} / \mathrm{CNTs}$, scan rate: $50 \mathrm{mV} \cdot \mathrm{s}^{-1}$; (d) production of $\cdot \mathrm{OH}$ from different nanocatalysts by ESR; (e) proposed mechanism in AuPd/CNTs catalytic degradation of organic pollutants ${ }^{63}$ ESR: electron spin resonance. color online

酸性条件下实现了对 TCE 的高效降解 ${ }^{67}$ 。电催化过 程中产生的氧化性中间产物(氧气、过氧化氢和羟 基自由基等)由于硫酸盐的氧化, 为 $\mathrm{SO}_{4}^{-}$・形成提供 了物质保证, 进而确保 TCE 从地下水中的高效去 除。基于这些研究基础, Yuan 课题组在地下水有 机无机复合污染的多效电催化处理研究也取得了 进展。他们在沿地下水流方向顺次设置穿孔式铁 阳极、多空铜阴极及复合氧化物阳极, 当含有 TCE、硝酸盐、重金属铬的地下水流经电极区域 时, 在适宜 $\mathrm{pH}$ 、高电流 $(>45 \mathrm{~mA})$ 下通过直接电极 反应或与电极反应产物等发生一系列氧化还原反 应, 实现 TCE、硝酸盐、铬酸盐、砷酸和硒酸盐 的高效转化, 为电催化氧化还原转化及去除水中 复合污染物的研究奠定了理论基础(见图 7(d) $)^{68}$ 。

\section{2 电催化水体重金属转化与去除}

水体重金属复合污染的问题由来已久, 严重 威胁着人类的饮水安全。铬、砷、铅等重金属离 子 ${ }^{69,70}$ 对地下水的污染严重威胁着众多发达及发展 中国家地下水资源环境 ${ }^{71,72}$ 。已有研究采用电还原 方法对 $\mathrm{Cr}(\mathrm{VI})$ 进行还原去除 ${ }^{73}$, 但直接的电还原效 率和过高的能耗显然不尽如人意。Qian 等 ${ }^{74}$ 利用 $\mathrm{Pd} / \mathrm{Al}_{2} \mathrm{O}_{3}$ 催化电极表面高效氢化作用, 通过阴、阳
极反应过程调控还原 $\mathrm{Cr}(\mathrm{VI})$ 的 $\mathrm{pH}$ 环境, 实现了在 酸性条件下使 $5 \mathrm{mg} \cdot \mathrm{L}^{-1} \mathrm{Cr}(\mathrm{VI})$ 在 $30 \mathrm{~min}$ 内还原为 $\mathrm{Cr}(\mathrm{III})$ 的效率达到 $95 \%$ 。研究认为 $\mathrm{Pd}$ 基催化电极表 面形成的氢原子对 $\mathrm{Cr}(\mathrm{VI})$ 的还原起到了重要作用, 也暗示了生成的 $\mathrm{H}_{2} \mathrm{O}_{2}$ 对 $\mathrm{Cr}(\mathrm{VI})$ 还原也具有一定的 贡献。这个工作对水体铬重金属的污染的去除提 供较为新颖的思路, 也扩展了 $\mathrm{Pd}$ 基催化电极的水 处理应用范围。同时含有砷、铭等重金属离子的 酸性废水的处理是一直困扰着研究人员的难点问 题。其原因主要是, 一方面砷的去除需要将剧毒 性三价砷氧化成五价砷, 再使其沉淀去除 ${ }^{75}$; 另一 方面, 需要利用还原剂 $\left(\mathrm{Na}_{2} \mathrm{~S}_{2} \mathrm{O}_{3} 、 \mathrm{Na}_{2} \mathrm{SO}_{3}\right.$ 或 $\mathrm{FeSO}_{4}$ 等)将六价铬还原为三价铬, 使铬生成 $\mathrm{Cr}(\mathrm{OH})_{3}$ 沉淀 从水中去除 ${ }^{76}$ 。同时实现二者的氧化与还原过程的 研究鲜有报道。Sun 等 ${ }^{77}$ 在利用抗坏血酸还原法制 备了粒径更小的 AuPd 合金, 在 CNTs 基底上制备 成分布更均匀的复合电极(图 8(a))。结果证明, 该 电极具有更好的原位电催化合成 $\mathrm{H}_{2} \mathrm{O}_{2}$ 的性能, $\mathrm{H}_{2} \mathrm{O}_{2}$ 生成率达到 $85 \%$ 以上, 首次实现 $\mathrm{Cr}^{6+}$ 还原为 $\mathrm{Cr}^{3+}$ 和 $\mathrm{As}^{3+}$ 向 $\mathrm{As}^{5+}$ 的高效协同转化(图 8(b))。此外, 制备的 $\mathrm{AuPd} / \mathrm{CNTs}$ 提供了较多的 $\mathrm{AuPd}(111)$ 活性晶 面, 使其具有高效催化氧气依照二电子还原路 

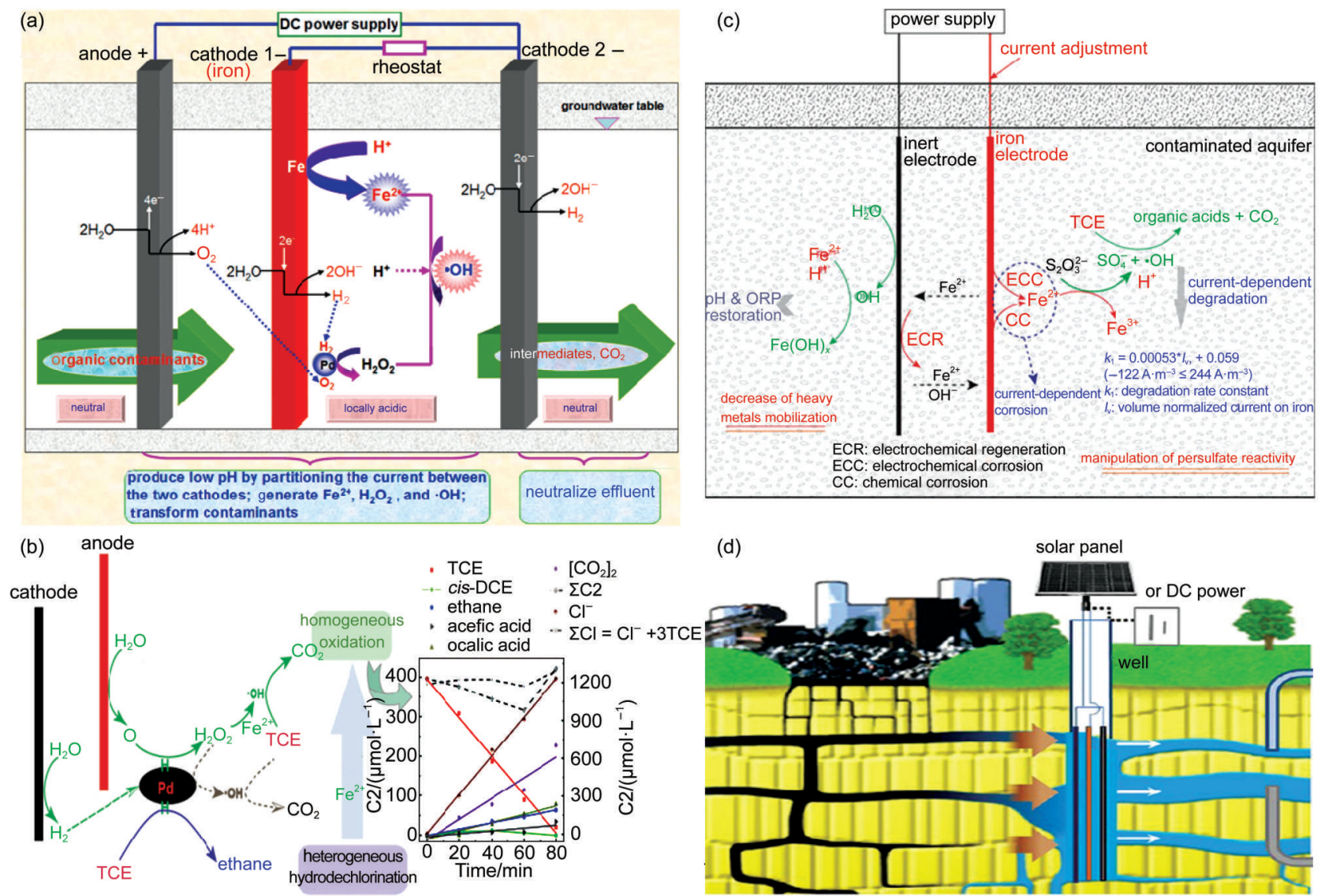

图 7 (a) 电化学阳极溶解 $\mathrm{Fe}^{2+}$ 对 $\mathrm{Pd}$ 电催化转化处理地下水中甲基叔丁醚的机制图 ${ }^{65}$; (b) $\mathrm{Fe}^{2+}$ 对 $\mathrm{Pd}$ 电催化三氯乙烯 降解作用的影响机制图 ${ }^{62}$; (c)过硫酸盐强化电催化三氯乙烯降解作用机制图 ${ }^{66}$;

(d)电催化辅助多效反应带处理地下水中三氯乙烯及硝酸盐机制图 ${ }^{68}$

Fig.7 (a) Mechanisms for electrochemical generation of $\mathrm{Fe}^{2+}$ from an iron cathode for Pd-catalytic transformation of MTBE in groundwater ${ }^{65}$; (b) mechanisms for TCE degradation in the presence of $\mathrm{Fe}(\mathrm{II})^{62}$; (c) scheme of electrolytic manipulation of persulfate reactivity for trichloroethylene degradation ${ }^{66}$; (d) scheme of electrochemically induced dual reactive barriers for transformation of TCE and nitrate in groundwater ${ }^{68}$ MTBE: methyl tert-butyl ether; TCE: trichloroethylene

径, 使约 $97.2 \% \pm 2.4 \%$ 的 $\mathrm{Cr}^{6+}$ 还原和 $95.7 \% \pm 4 \%$ 的 $\mathrm{As}^{3+}$ 氧化。实验结果还发现, 铬的还原优先于砷的 氧化过程, 且这种共转化效率随电流增大、 $\mathrm{pH}$ 降 低而增强。如图 8(c) 和 8(d) 所示, 中间产物 $\mathrm{Cr}(\mathrm{V})$ 和 $\mathrm{As}(\mathrm{IV})$ 及活性自由基 $\mathrm{HO}$ - 和 $\mathrm{O}_{2}^{*}$ 的电催化铬、砷 共转化的详细反应过程被解析, 并通过密度泛函 理论计算结果得到证实(见图 8(e))。这种 $\mathrm{AuPd}$ / CNTs 催化电极也具有较好的催化稳定性, 其可稳 定实现 10 次以上的铬砷共转化过程 ${ }^{77}$ 。此外, Qian 等 ${ }^{78}$ 也基于点催化氧还原作用实现了在中性及碱性 条件下对地下水中痕量 $\mathrm{As}(\mathrm{III})$ 的氧化。研究证明, 地下水孔隙水发育及富含 $\mathrm{HCO}_{3}^{-} 、 \mathrm{Ca}^{2+} 、 \mathrm{Mg}^{2+}$ 和腐 殖酸条件有利于 $\mathrm{As}(\mathrm{III})$ 氧化转化。他们又通过电催 化并辅助 $\mathrm{Fe}(\mathrm{II})$ 共沉淀作用使地下水中 $\mathrm{As}(\mathrm{III})$ 转化
为 $\mathrm{As}(\mathrm{V})$ 后去除, 实现了原位对含高浓度 $\mathrm{As}(\mathrm{III})$ 地 下水的高效处理, 达到净化地下水的目的。

\section{3 电催化水中有机污染物还原去除}

$\mathrm{Qu}$ 课题组 ${ }^{79,80}$ 针对饮用水中无机盐类和微量含 氯或其他卤素有机物的去除进行研究。由于此类 有机物较难被氧化去除, 将其还原脱卤后再去除 是明智选择。首先, 通过共沉淀法制备了 $\mathrm{Pd} / \mathrm{Sn}$ 电 极并成功实现了对水中硝酸盐还原去除 ${ }^{79}$ 。接着采 用电沉积方法制备了 $\mathrm{Pd} / \mathrm{Fe}$ 负载碳纸电极 $(\mathrm{Pd} / \mathrm{Fe}$ $\mathrm{C})^{80}$ 。结果表明, 该电极在氯乙酸的还原去除性能 上优于传统电极, 在 $\mathrm{Pd}: \mathrm{Fe}$ 摩尔比为 2 、电位 为 $-1.5 \mathrm{~V}$ 及 $\mathrm{pH}=3$ 条件下, $600 \mu \mathrm{g} \cdot \mathrm{L}^{-1}$ 三氯乙酸在 $20 \mathrm{~min}$ 内即可被完全还原去除。三氯乙酸的还原速 率最快, 二氯乙酸次之, 而一氯乙酸却无法直接 

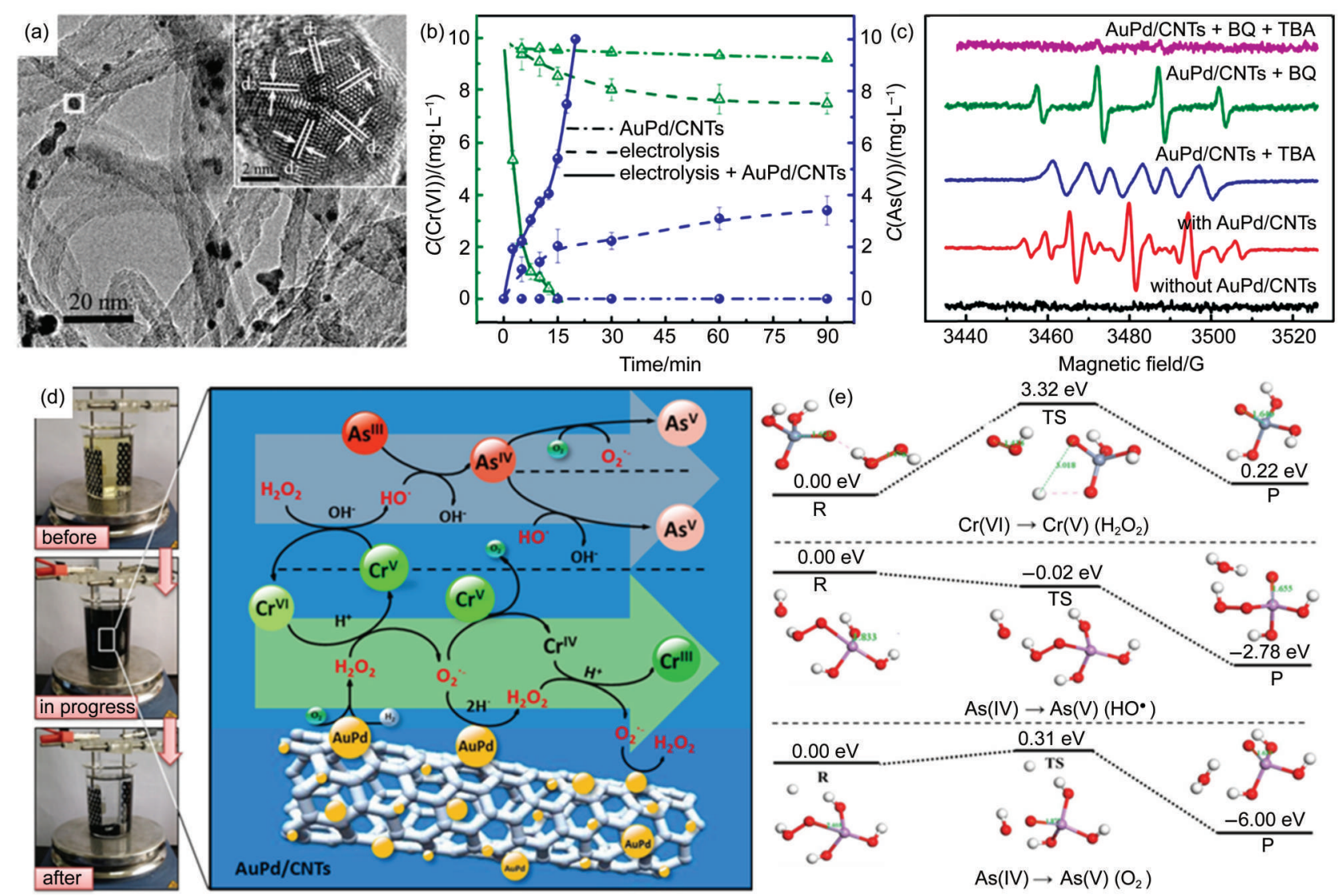

图 8 (a) AuPd/CNTs 电极的透射电镜图片; (b)AuPd/CNT 存在时砷、铬共转化效率; (c)不同催化体系中自由基的 ESR 谱 图; (d) 基于 AuPd/CNTs 电催化砷、铬共转化的机制; (e)电催化砷、铬共转化过程中主要存在反应的密度泛函计算结果 ${ }^{77}$

Fig.8 (a) TEM image of AuPd/CNTs; (b) redox conversion of $\mathrm{Cr}(\mathrm{VI})$ and $\mathrm{As}(\mathrm{III})$ in the presence of $\mathrm{AuPd} / \mathrm{CNTs}$; (c) hyperfine EPR spectra of active free radicals detected in different electrocatalysis conditions; (d) proposed mechanism of simultaneous transformation of $\mathrm{Cr}(\mathrm{VI})$ and $\mathrm{As}(\mathrm{III})$ under $\mathrm{AuPd} / \mathrm{CNTs}$ electrocatalysis; (e) density functional theory calculations of the intermediate reactions in the redox conversion of $\mathrm{Cr}(\mathrm{VI})$ and $\mathrm{As}(\mathrm{III})^{77}$

被电还原。研究还发现, 氯乙酸可在 $\mathrm{Pd} / \mathrm{Fe}-\mathrm{C}$ 作用 下有效脱氯, 且在该过程中电催化剂元素价态基 本保持不变, 可保持长效活性。通过同样方式将 Pd 颗粒沉积在颗粒活性炭 (GAC) 上, 制备了 Pd$\mathrm{GAC}$ 颗粒电极并填充在阴阳两极之间, 在三电极 体系中对氯乙酸进行电还原处理 ${ }^{81}$ 。研究表明, 在 电流密度 $0.3 \mathrm{~mA} \cdot \mathrm{cm}^{-2}$ 条件下, $120 \mu \mathrm{g} \cdot \mathrm{L}^{-1}$ 的氯乙 酸可在 $20 \mathrm{~min}$ 内还原去除 $50 \%$, 此时电子传递效 率与氯乙酸的弥散作用是其主要影响因素。当电 流继续增大至 $0.6 \mathrm{~mA} \cdot \mathrm{cm}^{-2}$ 、氯乙酸初始浓度增加 至 $600 \mu \mathrm{g} \cdot \mathrm{L}^{-1}$ 时, 控制其还原的主要因素仅为弥散 作用。随后的研究证明, $\mathrm{Pd} / \mathrm{rGO} / \mathrm{CFP}^{82}$ 和 $(\mathrm{Pd} /$ modified-C $)^{83}$ 可在溴酸盐的电催化还原中发挥重要作 用。 $\mathrm{Mao}$ 等对 $\mathrm{Pd} / \mathrm{C}$ 和 $\mathrm{Pd} / \mathrm{GAC}$ 催化剂进行了改进, 用石墨烯进行修饰, 分别增强了这两种 $\mathrm{Pd}$ 基催化 剂对澳酸盐的电催化还原去除能力。石墨烯修饰 的 $\mathrm{Pd}$ 基催化剂促进了 $\mathrm{H}_{2} \mathrm{O}$ 在其表面的还原, 大量
的产物原子氢加速了 $\mathrm{BrO}_{3}^{-}$的还原。电子自旋共振 结果证实了关键中间产物原子氢的产生及作用, 推动了对 $\mathrm{Pd}$ 基催化剂电催化还原机制的研究, 揭 示了 $\mathrm{Pd}$ 基催化剂还原氯乙酸和硝酸盐的机制和原 理。除了饮用水, 电催化还原转化作用在地下水 有机污染的去除等方面也独具优势。Yuan 等 ${ }^{84}$ 制备 了 $\mathrm{Pd}$ 负载于表面部分氧化形成 $\mathrm{TiO}_{2}$ 的 $\mathrm{Ti}$ 片 $(\mathrm{Pd}-\mathrm{Ti} /$ $\mathrm{TiO}_{2} \mathrm{NTs}$ ) 阴极, 可高效将水中的三氯乙烯还原为乙 烷。结果表明, 在施加电流 $100 \mathrm{~mA}$ 、中性 $\mathrm{pH}$ 下, $21 \mathrm{mg} \cdot \mathrm{L}^{-1}$ 的 TCE 在 $120 \mathrm{~min}$ 内的去除率可达 $91 \%$ 。 TCE 的去除在一定程度上受水中存在的氯化 物与重碳酸盐的影响, 但硫化物与硫酸盐会显著 减弱 TCE 的还原去除。当施加电流逐渐增大时, $\mathrm{Pd}$ 基阴极的催化氢化作用对 TCE 还原去除的贡献 逐渐增加。

\section{4 结论与展望}


$\operatorname{Pd}$ 催化电极已在水处理领域中得到了广泛的 应用, 其在应对水中难降解污染物的处理及处置 方面表现出显著优势, 主要表现在: (i) 基于 $\mathrm{Pd}$ 基 催化电极催化氧气还原产生 $\mathrm{H}_{2} \mathrm{O}_{2}$ 性能, 在 $\mathrm{Fe}^{2+}$ 存在 下间接发生催化氧化反应, 以降解水中有机污染 物, 实现水质的净化; (ii) 利用 $\mathrm{Pd}$ 基催化电极催化 氢化性能, 实现对水中有机污染物、无机盐的还 原转化, 降低水中目标污染物浓度, 使其转化为 低毒性、易处理的产物, 进而从水中彻底去除, 达到净水目的; 再者, 同时利用 Pd 基催化电极的 氧还原及催化氢化性能, 对水中重金属离子进行 氧化、还原转化, 进一步实现对水中重金属离子 的去除。虽然 Pd 基催化电极在环境水处理领域表 现出较好的应用前景, 但其还需要在如下方面开 展有更深入的研究:

(1) 从原子层面认识催化反应机理。电催化氧 气还原反应具有复杂的动力学过程, 存在介于二 到四电子间的还原反应路径。如何调控电极组成 与结构及其催化性能, 减少副反应发生, 将大幅 提高电流效率而降低催化剂用量和成本。目前, 仍然不能准确在原子层面上把控其反应机制。虽 然, 部分电子密度泛函理论的计算结果向人们提 供了氧气还原过程中可能存在的中间产物, 并预 测了催化剂分子结构中活性催化位点。由于缺乏 可佐证的实验数据或有效的表征手段, 对活性位 点的认识和理解还受限。并且, 随着近年来新型 催化剂组成与结构越趋复杂, 认识催化活性位点 的需求更为迫切。因此, 将最新发展的先进表征 技术和科学的理论计算方法相融合, 对于揭示电 催化反应过程机制至关重要。

(2) 新型可持续、高效催化电极的研制。Pd优 越的催化活性已得到广泛认可, 其在某些催化领 域已经表现出取代 Pt 的一定优势。但是, 相比较 于过渡金属来说, 相对高的价格及稀缺性使得寻 求更好的可替代元素成为明智的选择。研究表 明, 钴 ${ }^{85}$ 、铜 ${ }^{86,87}$ 、钛 ${ }^{88,89}$ 、铇 ${ }^{90}$ 、锌 ${ }^{91}$ 等过渡金属氧 化物、合金、矿物等在催化氧气还原方面有着潜 在的优势。碳基纳米材料(如碳纳米管、石墨烯等) 具有优异的结构特性、稳定性、热传导性及导电 性, 可作为复合电极的良好基底材料。选择无毒 害、绿色环保、性能优良的复合电催化剂用于催 化净水领域将更具现实意义。

(3) 电催化材料的规模化生产及其电化学水处
理工程的实际应用。从实验室研究转向实际生 产, 制备的纳米电极需要经历实际加工过程中存 在的诸如催化剂载量控制、粘合剂选择、合成压 力、温度不精准、活化时间不均等诸多因素的影 响。在实际应用方面, 有些研究已经指出了一些 纳米材料的潜在环境毒理学危害 ${ }^{22,93}$ 。虽然争议依 旧存在, 但从其实际水处理应用角度考虑, 催化 剂本身的生态毒性效应还是需要引起足够重视。 此外, 在水处理过程中, 催化剂的长效催化稳定 性和耐受性能否经受复杂水质的考验都需要大量 的基础科学研究做支撑。

相信在未来, 随着理论体系日趋完善、新型 材料的不断涌现, 可用于水处理的电催化剂的生 产及应用问题会逐步得到解决。新型催化电极的 研制与应用必将在二十一世纪水处理技术革新的 浪潮中占据重要一席。

\section{References}

(1) Shao, M. H.; Chang, Q. W.; Dodelet, J. P.; Chenitz, R. Chem. Rev. 2016, 116, 3594. doi: 10.1021/acs.chemrev.5b00462

(2) Liu, M. M.; Zhang, R. Z.; Chen, W. Chem. Rev. 2014, 114, 5117. doi: 10.1021/cr400523y

(3) Chlistunoff, J. J. Phys. Chem. C 2011, 115, 6496. doi: 10.1021/ jp108350t

(4) Levy, N.; Mahammed, A.; Kosa, M.; Major, D. T.; Gross, Z.; Elbaz, L. Angew. Chem. Int. Ed. 2015, 54, 14080. doi: 10.1002/ anie. 201505236

(5) Zheng, Y. R.; Gao, M. R.; Li, H. H.; Gao, Q.; Arshad, M. N.; Albar, H. A.; Sobahi, T. R.; Yu, S. H. Sci. China. Mater. 2015, 58, 179. doi: 10.1007/s40843-015-0034-5

(6) Brillas, E.; Sirés, I.; Oturan, M. A. Chem. Rev. 2009, 109, 6570. doi: 10.1021/cr900136g

(7) Luo, L. X.; Shen, S. Y.; Zhu, F. J.; Zhang, J. L. Acta Phys. -Chim. Sin. 2016, 32, 337. [罗柳轩, 沈水云, 朱风鹃, 章俊良. 物理化学学报, 2016, 32, 337.] doi: 10.3866/PKU. WHXB201509144

(8) Chen, A.; Ostrom, C. Chem. Rev. 2015, 115, 11999. doi: 10.1021/acs.chemrev.5b00324

(9) Zhang, H.; Jin, M.; Xiong, Y.; Lim, B.; Xia, Y. Accounts Chem. Res. 2013, 46, 1783. doi: 10.1021/ar300209w

(10) Zhu, C.; Zeng, J.; Lu, P.; Chlistunoff, J.; Gu, Z.; Xia, Y. Chemistry 2013, 19, 5127. doi: 10.1002/chem.201203787

(11) Yarulin, A. E.; Crespo-Quesada, R. M.; Egorova, E. V.; KiwiMinsker, L. L. Kinet. Catal. 2012, 53, 253. doi: 10.1134/ s0023158412020152

(12) Jin, M.; Zhang, H.; Xie, Z.; Xia, Y. Energ. Environ Sci. 2012, 5 , 6352. doi: 10.1039/C2EE02866B

(13) Liu, L.; Yoo, S. H.; Lee, S. A.; Park, S. Nano Lett. 2011, 11, 
3979. doi: $10.1021 / \mathrm{n} 1202332 \mathrm{x}$

(14) Tian, N.; Zhou, Z. Y.; Sun, S. G. Chem. Commun. 2009, 12, 1502. doi: 10.1039/b819751b

(15) Cui, C. H.; Yu, J. W.; Li, H. H.; Gao, M. R.; Liang, H. W.; Yu, S. H. ACS Nano 2011, 5, 4211. doi: 10.1021/nn2010602

(16) Adams, B. D.; Asmussen, R. M.; Ostrom, C. K.; Chen, A. C. J. Phys. Chem. C 2014, 118, 29903. doi: 10.1021/jp5095273

(17) Semaltianos, N. G.; Petkov, P.; Scholz, S.; Guetaz, L. J. Colloid Interface Sci. 2013, 402, 307. doi: 10.1016/j.jcis.2013.03.062

(18) Jukk, K.; Alexeyeva, N.; Sarapuu, A.; Ritslaid, P.; Kozlova, J.; Sammelselg, V.; Tammeveski, K. Int. J. Hydrog. Energy 2013, 38, 3614. doi: 10.1016/j.ijhydene.2013.01.062

(19) Adams, B. D.; Wu, G.; Nigro, S.; Chen, A. J. Am. Chem. Soc. 2009, 131, 6930. doi: 10.1021/ja901798u

(20) Gu, L.; Luo, N.; Miley, G. H. J. Power Sources 2007, 173, 77. doi: 10.1016/j.jpowsour.2007.05.005

(21) Spencer, J. A.; Rosenberg, S. G.; Barclay, M.; Wu, Y. C.; McElwee-White, L.; Fairbrother, D. H. Appl. Phys. A-Mater. 2014, 117, 1631. doi: 10.1007/s00339-014-8570-5

(22) Bhuvana, T.; Kulkarni, G. U. ACS Nano 2008, 2, 457. doi: $10.1021 / \mathrm{nn} 700372 \mathrm{~h}$

(23) Muniz-Miranda, M.; Gellini, C.; Canton, P.; Marsili, P.; Giorgetti, E. J. Alloy. Compd. 2014, 615, S352. doi: 10.1016/j. jallcom.2013.12.064

(24) Kim, J.; Reddy, D. A.; Ma, R.; Kim, T. K. Solid State Sci. 2014, 37, 96. doi: 10.1016/j.solidstatesciences.2014.09.005

(25) Kuai, L.; Yu, X.; Wang, S.; Sang, Y.; Geng, B. Langmuir 2012, 28, 7168. doi: 10.1021/1a300813z

(26) Xu, H.; Tong, Y. X.; Li, G. R. Acta Phys. -Chim. Sin. 2016, 32, 2171. [许 瀚, 童叶翔, 李高仁. 物理化学学报, 2016, 32, 2171.] doi: 10.3866/PKU.WHXB201606131

(27) Kang, W.; Li, H.; Yan, Y.; Xiao, P.; Zhu, L.; Tang, K.; Zhu, Y.; Qian, Y. J. Phys. Chem. C 2011, 115, 6250. doi: 10.1021/ jp111702s

(28) Ji, Y. G.; Wu, L.; Fan, Q. H. Acta Chim. Sin. 2014, 72, 798. [季 益刚, 吴 否, 范青华. 化学学报, 2014, 72, 798.] doi: 10.6023/ A14040325

(29) Chen, C. C.; Lin, C. L.; Chen, L. C. Electrochim. Acta 2015, 152, 408. doi:10.1016/j.electacta.2014.11.116

(30) Feng, Q. C.; Wang, W. Y.; Cheong, W. C.; Wang, D. S.; Peng, Q.; Li, J. P.; Chen, C.; Li, Y. D. Sci. China Mater. 2015, 58, 936. doi: 10.1007/s40843-015-0109-3

(31) Ye, J. S.; Chen, C. W.; Lee, C. L. Sensor. Actuat. B-Chem. 2015, 208, 569. doi:10.1016/j.snb.2014.11.091

(32) Ojani, R.; Abkar, Z.; Hasheminejad, E.; Raoof, J. B. Int. J. Hydrog. Energy 2014, 39, 7788. doi: 10.1016/j. ijhydene.2014.03.081

(33) Spencer, J. A.; Rosenberg, S. G.; Barclay, M.; Wu, Y. C.; McElwee-White, L.; Howard Fairbrother, D. Appl. Phys. A 2014, 117, 1631. doi: 10.1007/s00339-014-8570-5

(34) Barzola-Quiquia, J.; Schulze, S.; Esquinazi, P. Nanotechnology
2009, 20, 165704. doi: 10.1088/0957-4484/20/16/165704

(35) Torrisi, L.; Caridi, F.; Giuffrida, L. Nucl. Instrum. Meth. B 2010, 268, 2285. doi:10.1016/j.nimb.2010.03.029

(36) Mortazavi, S. Z.; Parvin, P.; Reyhani, A.; Golikand, A. N.; Mirershadi, S. J. Phys. Chem. C 2011, 115, 5049. doi: 10.1021/ jp1091224

(37) Sun, L. M.; Zhang, C.; Bao, Y. R.; Li, H. X. J. Electrochem. 2014, 20, 56. [孙丽美, 张 铖, 包英荣, 李宏霞. 电化学, 2014, 20, 56.] doi: 10.13208/j.electrochem. 121110

(38) Nguyen, T. T.; Pan, C. J.; Liu, J. Y.; Chou, H. L.; Rick, J.; Su, W. N.; Hwang, B. J. J. Power Sources 2014, 251, 393. doi: 10.1016/ j.jpowsour.2013.11.084

(39) Lee, Y. W.; Kim, M.; Kim, Z. H.; Han, S. W. J. Am. Chem. Soc. 2009, 131, 17036. doi: 10.1021/ja905603p

(40) Xu, J.; Wilson, A. R.; Rathmell, A. R.; Howe, J.; Chi, M.; Wiley, B. J. ACS Nano 2011, 5, 6119. doi: 10.1021/nn201161m

(41) Choi, S.; Jeong, H.; Choi, K. H.; Song, J. Y.; Kim, J. ACS Applied Materials \& Interfaces 2014, 6, 3002. doi: 10.1021/ am405601g

(42) Yang, D.; Carpena-Núñez, J.; Fonseca, L. F.; Biaggi-Labiosa, A.; Hunter, G. W. Sci. Rep. 2014, 4, 3773. doi: 10.1038/ srep03773

(43) Robert, C.; M.; Evan, U.; Cao, G. Z. Sci. China Mater. 2015, 58, 715. doi: 10.1007/s40843-015-0084-8

(44) Plowman, B. J.; Bhargava, S. K.; O'Mullane, A. P. Analyst 2011, 136, 5107. doi: 10.1039/C1AN15657H

(45) Shih, Z. Y.; Wang, C. W.; Xu, G.; Chang, H. T. J. Mater. Chem. A 2013, 1, 4773. doi: 10.1039/C3TA01664A

(46) Yang, L.; Hu, C.; Wang, J.; Yang, Z.; Guo, Y.; Bai, Z.; Wang, K. Chem. Commun. 2011, 47, 8581. doi: 10.1039/C1CC12528A

(47) Cernohorsky, O.; Zdansky, K.; Zavadil, J.; Kacerovsky, P.; Piksova, K. Nanoscale Res. Lett. 2011, 6, 410. doi: 10.1186/ 1556-276X-6-410

(48) Choudhary, V. R.; Samanta, C.; Choudhary, T. V. Appl. Catal. AGen. 2006, 308, 128. doi: 10.1016/j.apcata.2006.04.010

(49) Choudhary, V. R.; Samanta, C.; Jana, P. Appl. Catal. A-Gen. 2007, 332, 70. doi: 10.1016/j.apcata.2007.08.004

(50) Deguchi, T.; Iwamoto, M. J. Phys. Chem. C 2013, 117, 18540 doi: $10.1021 /$ jp 4056297

(51) Li, J.; Ishihara, T.; Yoshizawa, K. J. Phys. Chem. C 2011, 115, 25359. doi: 10.1021/jp208118e

(52) Li, J.; Staykov, A.; Ishihara, T.; Yoshizawa, K. J. Phys. Chem. C 2011, 115, 7392. doi: 10.1021/jp1070456

(53) Li, G.; Han, J.; Wang, H.; Zhu, X.; Ge, Q. ACS Catal. 2015, 5, 2009. doi: 10.1021/cs501805y

(54) Sheth, P. A.; Neurock, M.; Smith, C. M. J. Phys. Chem. B 2003, 107, 2009. doi: 10.1021/jp021342p

(55) Ham, H. C.; Hwang, G. S.; Han, J.; Nam, S. W.; Lim, T. H. J. Phys. Chem. C 2009, 113, 12943. doi: 10.1021/jp9043545

(56) Huang, Y.; Ferhan, A. R.; Dandapat, A.; Yoon, C. S.; Song, J. E.; Cho, E. C.; Kim, D. H. J. Phys. Chem. C 2015, 119, 26164. 
doi: 10.1021/acs.jpcc.5b08423

(57) Jirkovský, J. S.; Panas, I.; Ahlberg, E.; Halasa, M.; Romani, S.; Schiffrin, D. J. J. Am. Chem. Soc. 2011, 133, 19432. doi: $10.1021 / \mathrm{ja} 206477 \mathrm{z}$

(58) Jin, X.; Dang, L.; Lohrman, J.; Subramaniam, B.; Ren, S.; Chaudhari, R. V. ACS Nano 2013, 7, 1309. doi: 10.1021/ $\mathrm{nn} 304820 \mathrm{v}$

(59) Verdaguer-Casadevall, A.; Deiana, D.; Karamad, M.; Siahrostami, S.; Malacrida, P.; Hansen, T. W.; Rossmeisl, J.; Chorkendorff, I.; Stephens, I. E. L. Nano Lett. 2014, 14, 1603. doi: $10.1021 / \mathrm{nl} 500037 \mathrm{x}$

(60) Yuan, S.; Fan, Y.; Zhang, Y.; Tong, M.; Liao, P. Environ. Sci. Technol. 2011, 45, 8514. doi: 10.1021/es2022939

(61) Luo, M.; Yuan, S.; Tong, M.; Liao, P.; Xie, W.; Xu, X. Water Res. 2014, 48, 190. doi:10.1016/j.watres.2013.09.029

(62) Yuan, S.; Mao, X.; Alshawabkeh, A. N. Environ. Sci. Technol. 2012, 46, 3398. doi: 10.1021/es204546u

(63) Sun, M.; Zhang, G.; Liu, Y.; Liu, H.; Qu, J.; Li, J. Chem. Eur. J. 2015, 21, 7611. doi: 10.1002/chem.201406676

(64) Qin, Y.; Sun, M.; Liu, H.; Qu, J. Electrochim. Acta 2015, 186, 328. doi:10.1016/j.electacta.2015.10.122

(65) Liao, P.; Yuan, S.; Chen, M.; Tong, M.; Xie, W.; Zhang, P. Environ. Sci. Technol. 2013, 47, 7918. doi: 10.1021/es401730s

(66) Yuan, S.; Liao, P.; Alshawabkeh, A. N. Environ. Sci. Technol. 2014, 48, 656. doi: 10.1021/es404535q

(67) Yuan, S.; Chen, M.; Mao, X.; Alshawabkeh, A. N. Water Res. 2013, 47, 269. doi:10.1016/j.watres.2012.10.009

(68) Mao, X.; Yuan, S.; Fallahpour, N.; Ciblak, A.; Howard, J.; Padilla, I.; Loch-Caruso, R.; Alshawabkeh, A. N. Environ. Sci. Technol. 2012, 46, 12003. doi: 10.1021/es301711a

(69) Demoisson, F.; Mullet, M.; Humbert, B. Environ. Sci. Technol. 2005, 39, 8747. doi: 10.1021/es050717s

(70) Falayi, T.; Ntuli, F. J. Ind. Eng. Chem. 2014, 20, 1285. doi: 10.1016/j.jiec.2013.07.007

(71) Kimbrough, D. E.; Cohen, Y.; Winer, A. M.; Creelman, L.; Mabuni, C. Crit. Rev. Env. Sci. Tec. 1999, 29, 1. doi: 10.1080/ 10643389991259164

(72) Rifkin, E.; Gwinn, P.; Bouwer, E. Environ. Sci. Technol. 2004, 38, 267A. doi: 10.1021/es040575m

(73) Scialdone, O.; D'Angelo, A.; De Lumè, E.; Galia, A. Electrochim. Acta 2014, 137, 258. doi:10.1016/j. jiec.2013.07.007

(74) Qian, A.; Liao, P.; Yuan, S.; Luo, M. S. Water Res. 2014, 48, 326. doi: 10.1016/j.watres.2013.09.043

(75) O'Day, P. A.; Vlassopoulos, D.; Root, R.; Rivera, N. Proc. Natl. Acad. Sci. U. S. A. 2004, 101, 13703. doi: 10.1073/ pnas. 0402775101

(76) Kim, D. H.; Bokare, A. D.; Koo, M. S.; Choi, W. Environ. Sci. Technol. 2015, 49, 3506. doi: 10.1021/es5056897

(77) Sun, M.; Zhang, G.; Qin, Y.; Cao, M.; Liu, Y.; Li, J.; Qu, J.; Liu, H. Environ. Sci. Technol. 2015, 49, 9289. doi: 10.1021/acs. est. 5 b01759

(78) Qian, A.; Yuan, S.; Zhang, P.; Tong, M. Environ. Sci. Technol. 2015, 49, 5689. doi: 10.1021/acs.est.5b00808

(79) Wan, D.; Liu, H.; Zhao, X.; Qu, J.; Xiao, S.; Hou, Y. J. Colloid Interface Sci. 2009, 332, 151. doi: 10.1016/j.cej.2014.10.081

(80) Li, A.; Zhao, X.; Hou, Y.; Liu, H.; Wu, L.; Qu, J. Appl. Catal. BEnviron. 2012, 111-112, 628. doi: 10.1016/j.apcatb.2011.11.016

(81) Zhao, X.; Li, A.; Mao, R.; Liu, H.; Qu, J. Water Res. 2014, 51, 134. doi:10.1016/j.watres.2013.12.028

(82) Mao, R.; Zhao, X.; Lan, H.; Liu, H.; Qu, J. Appl. Catal. BEnviron. 2014, 160-161, 179. doi: 10.1016/j.apcatb.2014.04.040

(83) Mao, R.; Zhao, X.; Qu, J. Electrochim. Acta 2014, 132, 151. doi: 10.1016/j.electacta.2014.03.170

(84) Xie, W.; Yuan, S.; Mao, X.; Hu, W.; Liao, P.; Tong, M.; Alshawabkeh, A. N. Water Res. 2013, 47, 3573. doi: 10.1016/j. watres.2013.04.004

(85) Wang, J.; Zhou, J.; Hu, Y.; Regier, T. Energ. Environ. Sci. 2013, 6, 926. doi: 10.1039/C2EE23844F

(86) Ai, Z.; Xiao, H.; Mei, T.; Liu, J.; Zhang, L.; Deng, K.; Qiu, J. J. Phys. Chem. C 2008, 112, 11929. doi: 10.1021/jp803243t

(87) Fu, L. J.; Gao, J.; Zhang, T.; Cao, Q.; Yang, L. C.; Wu, Y. P.; Holze, R. J. Power Sources 2007, 171, 904. doi: 10.1016/j. jpowsour.2007.05.099

(88) Zana, A.; Rüdiger, C.; Kunze-Liebhäuser, J.; Granozzi, G.; Reeler, N. E. A.; Vosch, T.; Kirkensgaard, J. J. K.; Arenz, M. Electrochim. Acta 2014, 139, 21. doi: 10.1016/j. electacta.2014.07.002

(89) Ishihara, A.; Tamura, M.; Ohgi, Y.; Matsumoto, M.; Matsuzawa, K.; Mitsushima, S.; Imai, H.; Ota, K. I. J. Phys. Chem. C 2013, 117, 18837. doi: 10.1021/jp405247m

(90) Liu, Y.; Shrestha, S.; Mustain, W. E. ACS Catal. 2012, 2, 456 doi: $10.1021 / \mathrm{cs} 200657 \mathrm{w}$

(91) Neelgund, G. M.; Oki, A.; Luo, Z. J. Colloid Interface Sci. 2014, 430, 257. doi: 10.1016/j.jcis.2014.04.053

(92) Ma, J.; Liu, R.; Wang, X.; Liu, Q.; Chen, Y.; Valle, R. P.; Zuo, Y. Y.; Xia, T.; Liu, S. ACS Nano 2015, 9, 10498. doi: 10.1021/ acsnano. $5 \mathrm{~b} 04751$

(93) Qu, G.; Liu, S.; Zhang, S.; Wang, L.; Wang, X.; Sun, B.; Yin, N.; Gao, X.; Xia, T.; Chen, J. J.; Jiang, G. B. ACS Nano 2013, 7, 5732. doi: $10.1021 / \mathrm{nn} 402330 \mathrm{~b}$ 